Itinéraires Itinéraires

Littérature, textes, cultures

2012-1 | 2012

Genres et avant-gardes

\title{
Le surréalisme entre efféminement et virilisation (1924-1933)
}

\section{Guillaume Bridet}

\section{(2) OpenEdition}

\section{Journals}

Édition électronique

URL : http://journals.openedition.org/itineraires/1273

DOI : 10.4000/itineraires. 1273

ISSN : 2427-920X

Éditeur

Pléiade

\section{Édition imprimée}

Date de publication : 1 septembre 2012

Pagination : 95-108

ISBN : 978-2-296-55776-5

ISSN : $2100-1340$

\section{Référence électronique}

Guillaume Bridet, "Le surréalisme entre efféminement et virilisation (1924-1933) », Itinéraires [En ligne], 2012-1 | 2012, mis en ligne le 01 septembre 2012, consulté le 19 avril 2019. URL : http:// journals.openedition.org/itineraires/1273; DOI : 10.4000/itineraires.1273

\section{(ब) $(\Theta \Theta$}

Itinéraires est mis à disposition selon les termes de la licence Creative Commons Attribution - Pas d'Utilisation Commerciale - Pas de Modification 4.0 International. 


\section{Le surréalisme entre efféminement et virilisation (1924-1933)}

\section{Abstract}

This article focuses on both the way surrealists consider women and women poets and artists, and the way they practice what can be qualified as feminine literary genres and lyricism. Their extreme ambivalence concerning women results from what they perceive as a risk of feminisation in a period, after World War I, where the model of military attitude remains very strong.

Keywords : surrealism, gender studies, literary genres, virilism, effeminacy Mots clés : surréalisme, études de genre, genres littéraires, virilisation, efféminement

«La pensée n'a pas de sexe; ne se reproduit pas. »

(André Breton et Paul Éluard)

On peut à juste titre considérer le surréalisme comme un mouvement qui a valorisé les marges : marges sociales, avec l'intérêt pour la culture populaire (cinéma, publicité, carte postale), pour les figures d'aliénés ou de criminels; marges culturelles, avec la valorisation des civilisations extra-occidentales; marges de genre enfin, avec l'idéalisation d'une figure de la femme à la fois inspiratrice et elle-même en contact avec les forces de l'inconscient. Mais cette reconnaissance, à la fois thématisée et liée à des enjeux esthétiques majeurs, a-t-elle conduit, sur le plan des pratiques, à une appropriation de la parole par les marges elles-mêmes et à une reconnaissance de cette parole par les membres du groupe surréaliste? La réponse semble à première vue contrastée. Si le groupe surréaliste est composé essentiellement d'individus de sexe masculin, issus de la bourgeoisie ou des classes moyennes et d'origine occidentale, d'autres, dont certains ont même acquis une grande notoriété, sont bien d'origine populaire ou viennent d'horizons nationaux qui ne se limitent pas à l'Europe. Concernant les femmes (pour ne rien dire des criminels et des 
aliénés), l'histoire du surréalisme est toutefois marquée par un phénomène étrange : une absence à peu près totale se renversant brusquement en une présence abondante. Tout bascule autour de la Seconde Guerre mondiale. Pourquoi cette occultation des femmes avant 1939 (malgré un frémissement dans la seconde moitié des années 1930, en particulier dans l'activité plastique) et pourquoi cet éclat après 1945, au point qu'on a pu présenter le surréalisme d'alors comme « le paradigme d'un art au féminin ${ }^{1} »$ ? On peut rendre compte de ce contraste par des explications de sociologie générale ou de sociologie plus spécifique au champ littéraire, mais aussi par un autre type d'explications qui tient au surréalisme lui-même, aux idées qu'il développe, aux pratiques qu'il met en œuvre, aux genres - mais le terme est impropre - qu'il valorise. De ce point de vue, il en va du surréalisme comme de la psychanalyse : témoins de leur temps, certes, et Freud pas davantage que Breton et ses compagnons n'échappent à une certaine pente misogyne, mais suffisamment novateurs aussi pour ouvrir des possibles et autoriser leur réalisation.

Concernant le surréalisme de l'avant-guerre, la revue constitue un bon point d'observation et, plus précisément, les deux revues majeures qui se succèdent sur une encablure de près de dix ans, La Révolution surréaliste de décembre 1924 à décembre 1929 et Le Surréalisme au service de la révolution de juillet 1930 à mai 1933. S'il faut prendre garde à ne pas surévaluer l'importance pour le groupe de telle ou telle contribution individuelle (un texte d'un collaborateur mineur n'est pas d'un grand poids face à une contribution d'Aragon ou a fortiori de Breton), considérer l'ensemble des numéros de ces deux revues permet d'éviter de fixer artificiellement une doxa collective à partir de quelques textes ou même de quelques auteurs, en l'occurrence de réduire le surréalisme aux positions réputées misogynes et homophobes de Breton, et d'occulter la réalité d'un mouvement beaucoup plus complexe et contradictoire.

Le premier constat que l'on doit faire est que « le mouvement surréaliste, au moins à ses débuts, est particulièrement peu ouvert au genre féminin ${ }^{2} \gg$. La très modeste contribution des femmes à nos deux revues (qui comptent chacune environ une soixantaine de contributeurs) en témoigne malgré une légère augmentation de l'une à l'autre : pour La Révolution surréaliste, on ne trouve que les noms de Renée Gauthier et Fanny Beznos; pour Le Surréalisme au service de la révolution, ceux de Symone Monnerot, Greta Knutson, Marie-Berthe Ernst, Gala Éluard et Valentine Hugo. Les femmes sont peu présentes; leur présence est aussi parfois occultée par le recours à de simples initiales, comme c'est le cas dans le premier numéro de

1. Catherine Gonnard et Élisabeth Lebovici, Femmes artistes, artistes femmes. Paris, de 1880 à nos jours, Paris, Hazan, 2007, p. 168.

2. Ibid., p. 170. 
La Révolution surréaliste, où les lettres $\mathrm{S}$. B. au bas d'un texte dissimulent sans doute Simone Breton ${ }^{3}$. Outre que ces femmes sont essentiellement des femmes de (le nom de la plupart d'entre elles le dit assez), leur faible degré d'implication dans le mouvement proprement dit est encore confirmé par le fait que, si l'on excepte le cas de Valentine Hugo qui apporte trois contributions, elles ne sont chacune présentes que dans un seul numéro de revue, elles participent très peu aux enquêtes collectives et elles ne signent pas (ou rarement) les déclarations collectives du groupe. Il faut ajouter que le faible nombre de leurs interventions n'est pas compensé par leur haute valeur symbolique. Le sommaire de ces deux revues ne compte aucun texte majeur écrit par une femme, que ce soit d'un point de vue théorique ou formel. Les femmes réalisent des œuvres inscrites dans des genres que l'on peut considérer comme mineurs : récit de rêve, texte automatique ou fabrication d'objets. Les poèmes ou les peintures restent peu nombreux. Si on reprend les analyses de Norbert Bandier sur le groupe surréaliste, cette participation modeste indique que les femmes appartiennent à cette catégorie d' « auteurs dépourvus de position réelle dans le champ littéraire ${ }^{4} \gg$. Seuls les surréalistes qui ont déjà un certain nombre de publications individuelles à leur actif sont a contrario en mesure de publier dans la revue, à côté des textes expérimentaux, des textes relevant d'une écriture identifiable comme poésie ou comme discours théorique.

Si les femmes apparaissent à tous égards modestement au sommaire des deux revues, elles sont en revanche abondamment évoquées et représentées, que ce soit dans les textes, dont elles constituent fréquemment l'objet central, et plus encore dans les différents dessins ou tableaux reproduits, au cœur desquels elles figurent, très souvent nues ou en petite tenue, parfois maîtresses d'école, religieuses ou prostituées, offertes au regard du lecteur. Ce contraste apparaît clairement dans le cahier photo du troisième numéro du Surréalisme au service de la révolution avec la photographie solarisée de Man Ray intitulée « Primat de la matière sur la pensée » et qui montre une femme sans identité précise - cet effacement est en lui-même significatif - nue, allongée, la tête à droite penchée vers le bas du tableau. Cette femme offerte au regard prend d'abord place à la suite des objets désirants que le lecteur a pu regarder dans les deux pages précédentes et dont deux étaient dus à des artistes de sexe féminin, Valentine Hugo et Gala Éluard. En écho avec l'ensemble du numéro ou même des numéros de la revue, la femme retrouve ainsi sa position d'objet - « matière » à photographier - plutôt que de sujet - usant éventuellement de sa «pensée ». Mais le «primat » de « la matière » sur « la pensée » peut encore s'entendre d'une autre manière qui souligne la nouveauté des moyens techniques dont use l'artiste : « la matière » désigne sous ce rapport la matérialité de la

3. S. B., «Textes surréalistes », La Révolution surréaliste [désormais abrégé LRS], n 1 , décembre 1924, p. 14.

4. Norbert Bandier, Sociologie du surréalisme. 1924-1929, Paris, La Dispute, 1999, p. 342. 
photographie, en l'occurrence les effets de solarisation, et « la pensée » renvoie à son thème, en l'occurrence la femme photographiée. La légende privilégie l'attention aux moyens de l'art sur l'objet qu'il représente, cet objet fût-il le plus désirable qui soit : une femme alanguie et nue. Il y a ici une poétique en même temps qu'une éthique du créateur, célibataire momentané de l'art, qui sacrifie la possession des biens de ce monde - de la femme - à la réalisation de sa vocation artistique ou à la contemplation esthétique. Ce que recouvre le corps féminin, c'est la présence d'une femme de tête; ce qu'elle révèle, c'est l'inventivité d'un artiste et sa capacité de sublimation. Tout est ainsi à sa place : les hommes sont au sommaire, les femmes sont un objet silencieux qui se laisse regarder.

D'une certaine manière, il ne faudrait pas faire d'anachronisme et cette faible présence des femmes ne doit pas étonner. Sauf exception - on pense en particulier au rôle qu'a pu jouer Rachilde au Mercure de France jusqu'en 1924, mais pour une large part cette revue appartient à une autre époque -, cette absence se vérifie également quand on se tourne vers les autres grandes revues des années 1920-1930 comme la NRF, Europe ou Les Cahiers du Sud. C'est tout le champ des revues littéraires, artistiques et intellectuelles de 1'entre-deux-guerres qui est concerné et on ne voit pas pourquoi le surréalisme échapperait à cette réalité globale d'une présence féminine raréfiée qui correspond au rôle que les femmes jouent dans la société et à un certain état du regard porté sur elles. Concernant plus spécialement le mouvement, certains indices ne trompent pas, qui indiquent un certain mépris, voire une véritable haine des femmes. La femme : voilà l'ennemi, dans ce qui ressemble véritablement à une guerre des sexes.

Premier fait marquant: on ne compte plus les propos misogynes dans nos deux revues. Ce qui relève du féminin est profondément rejeté, en particulier tout ce qui concerne la reproduction de l'espèce, dont les femmes constituent les dépositaires privilégiées : « chassez ces enfants, qui sont une perpétuelle insulte à l'amour ${ }^{5}$ ! », s' exclame Aragon. Le mouvement surréaliste cherche à se distinguer d'un certain type de féminité et des modes de pensée ou des pratiques qui lui sont attachés. Mépris des femmes? C'est encore trop peu. Les textes surréalistes révèlent un imaginaire marqué par une véritable sadisation du corps féminin. Le fait marquant n'est pas seulement la présence tutélaire de Sade dans Le Surréalisme au service de la révolution et sa défense contre toute tentative de lire son œuvre en terme psychopathologique, mais la cruauté exercée dans maints textes à l'égard d'un corps adoré mais violenté ou mis en pièce plus souvent qu'à son tour. Le thème de la virginité parcourt les deux revues, et il est souvent lié à un désir de viol globalement très présent (en particulier chez Artaud, Dali, Péret ou Giacometti). Le surréaliste aime à en mourir, il aime jusqu'à violer, mais il aime aussi à tuer ou à voir mourir. Ici, Desnos évoque une femme 
qui lui apparaît la nuit et qu'il envisage « de tuer [...] avec un poignard malais à longue lame »; là, il imagine une femme se tuant d'un coup de revolver dans le cœur et s'exclamant: « je t'offre la blessure béante de mon sexe et celle sanglante de mon cœur! $»^{6}$. Ce corps de femme mis à mal apparaît également dans les illustrations des deux revues, en particulier dans certains numéros de La Révolution surréaliste qui multiplient les représentations de corps féminins amputés des mains, des pieds, de la tête. Hommage à la beauté ? Sans aucun doute. Mais la femme telle que la représente l'iconographie surréaliste n'en demeure pas moins souvent un buste nu offert sans défense au regard et auquel a été retirée toute possibilité de préhension, de mouvement et de pensée.

Il faut bien sûr faire la part de la provocation érotique et morale dans tout ce matériel fantasmatique, mais il faut aussi noter que, si l'on se concentre sur la poésie et sauf exception, il semble bien que la femme soit l'ennemie du poète comme du révolutionnaire qu'entend être l'homme surréaliste. $\mathrm{La}$ chose apparait clairement dans l'article que le groupe consacre collectivement aux déboires conjugaux de Charlie Chaplin. Les surréalistes mettent d'abord en cause la femme de l'acteur pour une plainte qu'elle aurait déposée contre son mari, en particulier pour le fait qu'il l'aurait contrainte à des fellations, puis ils finissent par faire plus largement le procès de la femme mariée. Devenue "vache » et « couveuse mécanique », la femme de Chaplin n'est plus dès lors qu' " une de ces garces dont on fait dans tous les pays les bonnes mères, les bonnes sœurs, les bonnes femmes, ces pestes, ces parasites de tous les sentiments et de tous les amours $»^{7}$. La guerre conjugale des sexes se double en outre d'une guerre artistique entre la femme et le créateur. Autant elle se montre puritaine et finalement mal aimante, autant lui est un génie par ailleurs apôtre de l'amour authentique commandant de « laisser toute chose, et par exemple, au minimum, un foyer. Le monde avec ses biens légaux, la ménagère et les gosses appuyés par le gendarme, la caisse d'épargne ${ }^{8} \gg$. D'un côté, on trouve la femme, les enfants, le mariage, la société et la loi; de l'autre, le génie et ses camarades qui le soutiennent, une sexualité libérée et l'amour véritable.

Puisque la femme épouse et mère apparaît comme une alliée de la société qui cherche à briser la libre expression du génie, puisqu'elle est une ennemie du poète, il n'y a rien d'étonnant à ce qu'elle ne puisse réellement être poète elle-même. Dans les revues surréalistes, toute figure de femme qui prend un tant soit peu la plume est aussitôt brocardée, au mieux avec une ironie légère, au pire et le plus souvent avec une rare violence. Dans un article qu'il consacre à la SDN, Desnos évoque ainsi une « stupide femme

6. Respectivement Robert Desnos, «Journal d'une apparition », LRS, n 9-10, octobre 1927, p. 11, et « La baie de la faim », $L R S, \mathrm{n}^{\circ} 4$, juillet 1925, p. 18.

7. « Hands off love », LRS, n ${ }^{\circ}$ 9-10, octobre 1927, p. 3-4.

8. Ibid., p. 5. 
de lettres » en lutte contre la « littérature pornographique », puis il se livre à une attaque en règle contre « la vieille pucelle rancie »: " Admirable spectacle : une femme de lettres, aux seins tombants, discutant, avec quelle science, du crime de ces livres qui lui rappellent que voici longtemps déjà que sa décrépitude éloigne d'elle les amants vigoureux ! ${ }^{9}$. Cette femme, sans doute Anna de Noailles - seule femme de lettres à apparaître dans d'autres numéros de la revue et toujours soigneusement insultée - condense en elle tous les clichés attachés, au moins depuis le XIX ${ }^{\mathrm{e}}$ siècle - pensons à Baudelaire ou Barbey -, au lien entre littérature féminine et frustration sexuelle. Si la femme crée, ce ne peut être en effet que par compensation, et en particulier parce que les hommes se détournent d'elle et qu'il ne lui reste donc que cela - écrire - pour exalter ses humeurs et attirer l'attention.

Hostiles à l'égard des femmes, les surréalistes? La chose est indéniable et, en même temps, il n'est pas si loin, ce monde imaginé par Tzara dans lequel « les hommes ne parleront plus, tandis que les femmes chanteront ${ }^{10}$ ». S'il est bien en effet un mouvement ouvert au féminin - en tout cas à ce qu'il construit comme tel - c'est bien le surréalisme.

Premier aspect, le plus évident : les poètes surréalistes chantent la femme, sa beauté et l'amour qu'elle inspire. Certes, on pourrait affirmer - et la critique d'inspiration féministe ne s'en est pas privée, en particulier Xavière Gauthier dans son livre fondateur de 1971, Surréalisme et sexualité - qu'on retrouve là des figures répertoriées qui finalement sont toutes des figures de la mère, ce qui confère aux figures féminines une évidente puissance mais ne va pas sans impliquer une certaine ambivalence. Qu'elle construise l'image d'une femme enfant ou d'une femme nature, fleur, comète ou fruit, qu'elle soit bénéfique ou maléfique, fée ou sorcière, passante magnifique ou mante religieuse, la perspective surréaliste est essentiellement différentialiste et les métamorphoses auxquelles elle soumet la femme renvoient toutes plus ou moins à un éternel féminin qui n'est sans doute pas le mieux à même de permettre une émancipation réelle des femmes sur un plan pratique.

On peut toutefois être sensible à ce que la poésie surréaliste compte de poèmes d'amour, et le nom d'Éluard est sans doute celui qui est attaché le plus évidemment à cette promesse de bonheur que les femmes projettent sur la vie par le biais d'une érotisation généralisée et d'un blasonnement émerveillé du corps nu et variablement offert : la chevelure, la bouche, le sexe, les seins. Il faut ajouter que certains textes ouvrent des pistes plus originales que cette exaltation de la beauté féminine. Si on en reste au chapitre sexuel et en marge des déclarations conservatrices de certains membres du groupe, la liberté que manifestent les autres rejaillit aussi en

9. Robert Desnos, « Description d'une révolte prochaine », LRS, n 3, avril 1925, p. 26.

10. Tristan Tzara, « Grains et issues. Rêve expérimental », Le Surréalisme au service de la révolution [désormais abrégé $L S S R$ ], no 6 [mai 1933], p. 49. 
partie sur les femmes. La parole qui se libère est ainsi à l'occasion celle du désir féminin, comme lorsque Simone Breton formule ce vœu des plus directs : " Je souhaite que pendant sept jours vous aviez [sic] autant de sexes que de doigts à votre main droite ${ }^{11}$. » Loin de séparer la sphère des activités masculines de celle des activités féminines, le surréalisme a aussi, plus qu'aucun autre mouvement de son époque, mis à mal la dichotomie des représentations de l'homme et de la femme en ouvrant les portes de l'inconscient. Les exemples d'incertitude quant à l'identité sexuelle des protagonistes sont multiples, qui parsèment les numéros et qu'on trouve au détour de tel ou tel texte, au point qu'on peut véritablement parler de trouble dans le genre. Le corps masculin se féminise ou mieux encore s'indétermine, quand un personnage masculin inventé par Péret se perçoit avec « trois testicules dont un, celui du milieu, qui avait la forme, la couleur et les dimensions d'une framboise ${ }^{12} »$. Si le corps ou même l'allure de la femme se virilisent peu dans nos deux revues, en revanche, l'homme se transforme, et son désir aussi, qui se tourne à l'occasion vers son semblable (chez Leiris par exemple). Des poètes comme Pierre Yoyotte, mais aussi Crevel ou Dali de manière encore plus systématique, ouvrent véritablement la boîte à fantasmes et ils les identifient comme des productions de l'esprit n'impliquant nul raidissement viril et avec lesquelles on peut jouer.

Le propos prend même parfois une tonalité franchement féministe. Il ne convient certes pas de surévaluer l'importance des quelques pages qui mettent en avant des figures de femmes affranchies et favorables à la lutte révolutionnaire ${ }^{13}$ : elles sont rares, contredites par d'autres pages, et les auteurs qui les signent n'occupent pas une position de premier plan au sein du mouvement surréaliste dans ces années 1924-1933. Elles indiquent toutefois que le surréalisme peut supporter de tels propos et elles dessinent ainsi un avenir possible du mouvement, mieux même elles constituent les premiers signes d'une pratique poétique présente et surtout future. On peut en effet considérer que les surréalistes ont poussé très loin l'idéalisation de la muse, si loin qu'ils l'ont en grande partie transformée en une figure inédite presque autant inspirée elle-même qu'inspiratrice. On sait que pour les surréalistes, certains types de femmes connaissent une communication privilégiée avec les forces primitives de la vie et sont détentrices des secrets immémoriaux. Il y a les voyantes, les hystériques, et il y a Nadja, qui entraîne le poète dans une déambulation merveilleuse. Méprisées, maltraitées, voire enfermées, objets de la psychiatrie ou victimes de la police et des juges, ces figures de femmes incarnent des forces de résistance à la science officielle, elles annoncent le monde de demain, elles l'appellent même par leurs paroles et par leurs actes

11. S. B., « Textes surréalistes », op. cit.

12. Benjamin Péret, «L'amour des heures, la haine du poivre », $L R S, \mathrm{n}^{\circ}$ 3, avril 1925, p. 12.

13. Voir par exemple les poèmes de Raymond Queneau, Jacques Baron et Fanny Beznos, $L R S, \mathrm{n}^{\circ}$ 9-10, octobre 1927, p. 20-23. 
et elles constituent de ce point de vue des alliées précieuses du poète, mieux encore des modèles, comme l'enfant, comme le nègre, comme le fou. Elles proposent l'exemple à suivre d'un contre-art poétique qui vient subvertir toutes les valeurs esthétiques en place. Et il y a là une profonde nouveauté : autant la muse inspire puis reçoit passivement l'offrande du poète, autant la femme modèle est reconnue dans sa puissance créatrice. La créativité féminine est certes par nature entravée et elle ne trouve encore sa pleine réalisation que dans la parole poétique masculine. Breton le dit clairement concernant les voyantes : «Où réside votre seul tort, c'est dans l'acceptation de la scandaleuse condition qui vous est faite [...]. Mais cette passivité, toutes femmes que vous êtes, il en est temps, je vous adjure de vous en dépar$\operatorname{tir}^{14}$. » Il n'en reste pas moins qu'avec Nadja, la femme est dotée de qualités éminentes qui sont celles de l'artiste : une artiste à l'état brut, mal dégrossie et dont le poète doit finalement s'éloigner du fait de la tentation dangereuse qu'elle incarne, mais qui n'en témoigne pas moins de sa propre imprégnation féminine et qui annonce également le devenir artiste possible des femmes.

Femmes adorées ou haïes, femmes libérées ou aliénées, femmes inspirantes-inspirées ou relais de tous les conformismes : certains éléments permettant d'expliquer cette ambivalence tiennent sans doute à la créativité en général (je ne les évoquerai pas), d'autres aux traits spécifiques de la créativité surréaliste, d'autres encore à l'époque particulière dans laquelle elle prend place.

Le premier élément majeur réside dans le contexte de l'après-guerre. Dans la société française des années 1920-1930, l'heure n'est pas à la reconnaissance des femmes. Comme l'a bien montré André Rauch, la Grande Guerre les avait vues investir l'espace public devenu vacant après le départ de trois millions de soldats sur le front mais la tension est à présent évidente entre des femmes qui ont goûté à l'émancipation et des hommes qui attendent qu'elles retournent vers l'espace domestique afin qu'ils puissent retrouver leur place. « Pour la première fois sans doute, la perspective de l'égalité de droit mais aussi de fait se dessine concrètement : elle apparaît aussitôt comme une menace réelle ${ }^{15}$. ॥ La célébration patriotique devient dès lors une arme contre l'égalité des genres. On fait couramment des révoltes dadaïstes et surréalistes le fruit d'un rejet de la Première Guerre mondiale. Mais si la guerre est condamnée avec virulence par les surréalistes, elle n'en continue pas moins d'imposer dans le même temps un modèle de virilité nourri par la primauté qu'on accorde aux valeurs civiques de dévouement, de courage, de sacrifice et qu'il n'est pas si aisé de révoquer. Comment le poète pourrait-il être à la hauteur du soldat qu'il fut ou que fut son père? Comment la poésie pourrait-elle ne pas apparaître comme bibelot futile

14. André Breton, «Lettres aux voyantes », LRS, $\mathrm{n}^{\circ}$ 5, octobre 1925, p. 22.

15. André Rauch, L'Identité masculine à l'ombre des femmes. De la Grande Guerre à la Gay Pride, Paris, Hachette Littératures, 2004, p. 32. 
devant les 1,4 millions de morts que causa le conflit? La chose apparaît avec le plus de clarté dans une lettre que Jean Genbach écrit à Breton alors qu'il est interné en hôpital psychiatrique et dans laquelle il retranscrit les paroles d'un médecin neurologue de l'armée qui le menace :

Tu es anxieux, poète surréaliste, obsédé par le suicide. Tu n'as pas honte? $\mathrm{Tu}$ oses écrire des romans, toi qui ne sais même pas ce que c'est que le service militaire? Et tu es fils d'un officier mort à la guerre? Comment la République tolère-t-elle l'existence d'individus comme toi? [...] Vous ne sentez donc pas que vous gangrenez la société ${ }^{16}$ ?

Les surréalistes ne récusent pas cette pression morale, mais ils lui trouvent un nouvel objet, ils en déplacent le champ d'application. Il s'agit de lutter contre la «nouvelle religion [qui] s'est établie depuis la guerre : $[\ldots]$ celle de morts » qui furent en fait des «bœufs menés à l'abattoir ${ }^{17}$, victimes bien sûr mais aussi et surtout coupables, parce que, sans se révolter, ils se sont mis au service de la patrie, de l'argent et de la morale chrétienne. Et pour ce faire, il n'est pas d'autre solution que de promouvoir un héroïsme authentique qui remplace le faux héroïsme des années de guerre. Telle est la raison pour laquelle Poincaré, l'homme des cimetières, est une cible récurrente des surréalistes, comme les cérémonies en hommage aux morts de 14 et les monuments qui leur sont consacrés et dont un poète imagine l'épitaphe rythmée d'un peu patriotique refrain : «le doigt dans le trou du $\operatorname{cul}^{18} \gg$. La guerre doit se poursuivre mais sur un autre front, qui n'est plus la frontière extérieure avec l'Allemagne mais la frontière intérieure - véritable guerre civile - qui sépare les poètes en révolte des assis de toute sorte. $\mathrm{La}$ guerre est finie, mais elle laisse la place à une militarisation de la vie littéraire. Le thème ne va pas sans une certaine grandiloquence, comme à la fin du Second manifeste, où Breton condamne le poète à mourir au piloris de ses idées comme d'autres sont morts pour avoir déserté le combat :

Qu'il use, au mépris de toutes les prohibitions, de l'arme vengeresse de l'idée contre la bestialité de tous les êtres et de toutes les choses et qu'un jour, vaincu - mais vaincu seulement si le monde est monde - il accueille la décharge de ses tristes fusils comme un feu de salve ${ }^{19}$.

L'image guerrière étonne chez cet antimilitariste mais elle témoigne bien de l'ambivalence de Breton à l'égard des vertus viriles exaltées dans la figure du guerrier en armes (et du bolchevik intraitable) et elle assure la dignité d'une poésie que pourrait menacer une mollesse toute féminine.

Le risque d'un acte poétique féminisé et donc perçu comme dégradant constitue bien un second élément de réponse. Très généralement, il faut

16. Jean Genbach, «Correspondance », LRS, $\mathrm{n}^{\circ} 11$, mars 1928, p. 29-30.

17. Paul Éluard, « De l'usage des guerriers morts », $L R S, \mathrm{n}^{\circ}$ 6, mars 1926, p. 29.

18. Benjamin Péret, « Je ne mange pas de ce pain-là », $L R S, \mathrm{n}^{\circ} 12$, décembre 1929, p. 51.

19. André Breton, « Second manifeste du surréalisme », ibid., p. 17. 
d'abord souligner à quel point les soucis surréalistes - souci de l'amour physique, souci de l'amour sentiment, souci surtout de leur coexistence relèvent du genre féminin. En marge des revues pornographiques destinées généralement aux hommes et proposant sans plus de manière des photographies de femmes dénudées, les revues surréalistes anticipent à maints égards sur les magazines féminins de l'après-guerre. Pour le dire rapidement : leur éthique, à l'époque subversive, est presque devenue notre norme. Car que désirent-ils finalement? Contre les pères et les puissants, vieillards et bourgeois confondus, qui font des enfants avec leur femme et satisfont leurs désirs sexuels au bordel ou dans l'adultère, les hommes surréalistes s'appuient sur la psychanalyse pour réclamer la levée des tabous mais dans le cadre d'une vie de couple épanouie. Dans le même esprit, ils multiplient les déclarations qui peuvent apparaître comme d'une grande naïveté, en tous les cas d'un idéalisme assez peu de saison en ces années 1920-1930 durement marquées par la guerre et bientôt par la crise économique et les troubles politiques. Certaines déclarations sont d'un sentimentalisme échevelé : «Oui, l'amour d'une femme est à chaque instant ma seule raison de vivre. La société peut donc disparaître, l'humanité crever et la planète sauter, seul l'être que je désire compte pour moi ${ }^{20}$. » Que l'amour soit présenté comme l'unique bonheur de la vie et qu'un homme s'en remette au sentiment qu'une femme éprouve pour lui ne peut qu'inspirer la moquerie des hommes de l'époque, auxquels une éducation corsetée apprend, sinon à taire leurs sentiments, au moins à les exprimer avec réserve. Quand les surréalistes font sortir l'amour de la sphère strictement privée pour en faire la clé, soit de la connaissance, soit de l'avenir politique de l'humanité, on n'est pas loin de toucher le ridicule. Alors que vers les monuments aux morts se dirigent dignement les patriotes et les veuves, que les ouvriers en colère font grève et défilent le poing levé, que l'Action française et les fascistes énervés se liguent et font le coup de poing, les surréalistes accordent à l'amour une importance qu'il n'eut jamais, à l'amour entendu comme sentiment et comme plaisir physique, à l'amour comme force révolutionnaire - au risque de passer pour des midinettes (ce que Claudel ou Drieu La Rochelle ne manquent pas de leur signifier).

Le dernier élément à prendre en compte est que, pour une large part, la pratique poétique des surréalistes est fondée sur un lyrisme et se déploie dans des genres marqués par des traits essentiellement féminins. Breton et Éluard écrivent ainsi que « le lyrisme est le genre de poésie qui suppose la voix inactive », ils évoquent encore « un poème » qui « doit être une débâcle de l'intellect », et que dire de leur définition de la poésie comme "l'essai de représenter, ou de restituer, par des cris, des larmes, des caresses, des baisers, des soupirs, ou par des objets ces choses ou cette chose que tend obscurément d'exprimer le langage articulé, dans ce qu'il a d'apparence de vie ou de dessein supposé $»^{21}$ ? La raison et l'action traditionnellement

20. Jean Genbach, « Correspondance », op. cit., p. 30.

21. André Breton et Paul Éluard, « Notes sur la poésie », ibid., p. 53. 
identifiées comme masculines sont mises de côté au profit d'une passivité et d'un idéal d'expression physiologique qui sont au contraire considérés comme des attributs de la femme. Il est en outre tout à fait notable que le surréalisme se développe dans des genres mineurs, en particulier dans la mesure où il rejette le roman et où il met en avant une matière essentiellement personnelle. Or, ce choix est marqué traditionnellement et de manière souvent péjorative d'un signe féminin. Sans aller nécessairement jusqu'aux journaux intimes des adolescentes et aux correspondances entre femmes, qui sont des pratiques d'écriture devenues spécifiquement féminines dès le XVIII ${ }^{e}$ siècle, la critique cantonne régulièrement les femmes aux genres autoréférentiels. Une femme écrit-elle un roman? Ce n'est que confession masquée. Lorsqu'un critique de l'époque écrit à propos des femmes, qu'il juge « inhabiles dans le domaine de l'abstraction et de la fantaisie intellectuelle », que « la poésie des surréalistes et de leurs successeurs, qui n'est qu'un jeu cérébral et ne représente rien d'accessible aux sens, leur est [...] fermée $\gg^{22}$, il qualifie bien tout un aspect de l'entreprise surréaliste. Il occulte toutefois d'autres pratiques du mouvement, comme la correspondance, la poésie amoureuse et, plus largement, tous les genres autoréférentiels, qu'il considère lui-même comme essentiellement féminines. Rares sont en effet les écrits surréalistes dans lesquels l'auteur ne prend pas la parole en son nom pour parler de lui, sa sensibilité, ses sentiments, sa position dans le monde. "A l'un, le domaine de l'abstraction et des raisonnements suivis. À l'autre celui de l'émotion ${ }^{23}$. » Mais dans un tel partage des tâches littéraires entre hommes et femmes, où placer les surréalistes? À une époque où journaux intimes et correspondances féminines sont « partie prenante de la production d'identités individuelles et collectives des jeunes filles et des femmes ${ }^{24} »$, est-il possible d'imposer le souci de l'intime, l'émotion lyrique et les genres littéraires autoréférentiels comme des attributs virils? Dans le contexte de la guerre toute proche et de la crise économique, est-il possible de procéder (comme Leiris bientôt) à une héroïsation de l'intériorité et de la considérer comme un lieu d'affirmation sociale et politique?

Le souci de l'intimité du couple et l'inscription dans des genres marqués par le féminin exigent quoi qu'il en soit en réaction le maintien de la femme à la cuisine, à la maternité ou sa lecture pleurnicharde des courriers du cœur. Il exige surtout une métamorphose du poète sentimental et lyrique en un poète viril : en armes. Cette militarisation et cette inscription révolutionnaire de la poésie, cette volonté d'une poésie action n'est pas pour rien dans la récusation par les surréalistes de toute pensée en termes de genre littéraire. Le texte de Tzara intitulé « Essai sur la situation de la poésie » est sans doute le plus clair concernant ce point. Racontant

22. Jean Larnac, Histoire de la littérature féminine en France, Paris, Kra, 1929, p. 242.

23. Ibid., p. 278.

24. Michelle Zancarini-Fournel, Histoire des femmes en France. XIX $-X X^{e}$ siècles, Rennes, PUR, 2005, p. 223. 
l'histoire de la poésie depuis le romantisme, l'auteur considère que, si l'on excepte quelques poètes comme Rimbaud et Lautréamont, tous les autres, symbolistes inventeurs du vers libre ou Apollinaire promoteur des calligrammes et de la suppression de la ponctuation, ont commis la même erreur : confondre révolution formelle et révolution tout court. Tzara n'est pas moins sévère avec les futuristes :

\footnotetext{
Inutile de dire à quel point me laisse indifférent un nouveau contenu mis dans un vers antique ou une vieille pensée placée à la manière d'un cheveu sur la soupe sur une forme nouvelle, l'un entraînant l'autre ou inversement. Que la pensée nouvelle doive se forger une forme nouvelle... les futuristes sont encore les seuls à agiter vigoureusement leur creux cervelet sur l'importance de ces problèmes ${ }^{25}$.
}

Ce qui change avec Dada et avec le surréalisme, c'est que «la poésie tend à devenir une activité de l'esprit. Elle tend à nier la poésie-moyen d'expression [...] en se dégageant du langage ou de sa forme ${ }^{26} \gg$. Tout l'effort est ici d'inventer, non pas des genres (définis par leur forme), mais des pratiques permettant de faciliter l'activité spontanée de l'esprit. Le récit des rêves, l'écriture automatique, le cadavre exquis, le collage, les diverses expérimentations et jeux de société partagés par les surréalistes, telle ou telle invention propre à un artiste comme la paranoïa-critique de Dali ou le frottage de Max Ernst, toutes ces procédures ont pour but de mettre à mal l'édifice de la raison et de la réalité qu'elle construit, pour permettre une subversion de la vie et une révolution de la société. Ni Tzara, ni Breton, ni aucun surréaliste ne pense la poésie en termes de genre littéraire. Ils la pensent en termes d'action de la révolte sur les mots et d'action des mots sur les choses. Le mot n'est ni un début ni une fin, il est un produit et un moyen; le mot est une arme contre la famille, la patrie, la religion et la bourgeoisie. Comme l'explique Breton en filant une image encore une fois proprement militaire renvoyant au déferlement des barbares dans les steppes d'Asie, il s'agit de libérer des « hordes de mots littéralement déchaînés » : « Elles pénétreront sans hâte, à coup sûr, dans les petites villes idiotes de la littérature qui s'enseigne encore et, confondant sans peine ici les bas et les hauts quartiers, elles feront posément une belle consommation de tourelles $»^{27}$. Le critère qui doit présider à l'évaluation d'une œuvre plastique ou poétique n'est pas sa beauté - soumise à telle ou telle norme, que les surréalistes brocardent par leur mauvais goût -, c'est le refus de l'ordre établi, la volonté de ruiner les valeurs en cours ou d'en introduire de nouvelles, l'intention subversive.

C'est dans ce cadre qu'il faut comprendre la violence des textes surréalistes. En insultant, en menaçant, en appelant au meurtre, on fait preuve de

25. Tristan Tzara, « Essai sur la situation de la poésie », LSSR, n 4 [décembre 1931], p. 17.

26. Ibid., p. 18.

27. André Breton, « Second manifeste du surréalisme », op. cit., p. 9. 
virilité, alors qu'on n'est plus ou pas sur le champ de bataille et qu'on laisse par ailleurs ouvertes les vannes d'un lyrisme sentimental; alors qu'on se livre à une activité poétique, on fait la preuve qu'on peut encore, qu'on peut aussi être un homme - un vrai.

À côté de l'expression triviale de la puissance sexuelle, finalement plutôt rare, sans doute parce qu'elle serait trop directe, les surréalistes préfèrent d'abord l'insulte. L'insulte essaime rarement dans le genre du poème - avec toutefois une exception répétée pour Péret qui truffe ses textes, quels qu'ils soient, d'insultes particulièrement triviales, du type de celles qu'il accole au nom d'Adolphe Thiers, à la fois « ventre de merde », « pieds de cochon » et « couilles [...] de papier mâché $»^{28}$. Elle se retrouve de manière privilégiée dans certains types de textes : lettres, manifestes, revues de presse et comptes rendus d'ouvrages, soit tous les genres dans lesquels il est question de se prononcer directement en tenant un discours sur les institutions honnies que sont l'armée, la police et l'Église, ou sur les contemporains, le plus souvent des contemporains haïs (parfois d'anciens membres du groupe). Toutes les ressources y passent : scatologie, animalisation et surtout sexualisation. Sous ce rapport, il faut noter une très nette homophobie - et pas seulement chez Breton - puisque la pédérastie est très souvent mentionnée à des fins polémiques. De l'insulte, il n'est pas rare que l'on passe à la menace physique. Outre que l'on ne compte pas les crachats jetés à la figure de tel ou tel adversaire, les envies homicides, voire les appels au meurtre sont nombreux. Certains sont d'ordre général et ne visent pas un individu précis mais les policiers, les prêtres, les psychiatres dans leur ensemble. D'autres sont plus circonstanciés. Une des cibles privilégiées des poètes surréalistes est Jean Cocteau - de ce point de vue double masculin d'Anna de Noailles - immanquablement associé à la pédérastie et qui suscite une véritable haine. «Et puis, sans rougir, car nous parviendrons bien à l'abattre comme une bête "puante", prononçons le nom de Jean Cocteau $^{29} »$, lance ainsi le doux Paul Éluard. Les menaces écrites entraînent parfois des passages à l'acte - modestes, tout de même. C'est le cas tout particulièrement dans une anecdote qui met en scène Aragon se rendant en juin 1930 chez un critique littéraire de L'Humanité ayant dit du mal de Majakowsky [sic] pour le rosser. L'homme se cache d'abord derrière sa femme et Aragon fait passer la vaisselle par la fenêtre. La police intervient mais elle ne met pas fin à l'agression, puisque le poète trouve encore le moyen de mettre son poing dans la figure du critique ${ }^{30}$. Le surréaliste se donne ici le beau rôle : on le voit, il a agi comme un poète - un vrai.

28. Benjamin Péret, « Je ne mange pas de ce pain-là », op. cit., p. 51.

29. P. E., "Le cas Lautréamont d'après le "Disque vert"», $L R S, \mathrm{n}^{\circ}$ 6, mars 1926, p. 3.

30. Voir «Un insulteur de Majakowsky reçoit une visite désagréable », LSSR, $\mathrm{n}^{\circ} 1$ [juillet 1930], p. 21-22. 
Le surréalisme des années 1924-1933 impose ainsi la nécessité de penser ensemble, dans un même système en mouvement et non comme des essences, le masculin, le féminin et leur négociation permanente. Au-delà des individualités, des poètes et des textes, il marque le temps, inachevé, d'une reconnaissance des attributs de la féminité dans le processus poétique et d'une féminisation (relative) de l'homme poète. La réaction surréaliste à la virilité exaltée par la guerre réside en effet pour partie dans une exaltation opposée et réactive du féminin ou de ce qui est perçu et construit comme tel : proximité avec la nature, attention à soi et aux autres, ouverture au mystère. Pour une autre partie toutefois, elle consiste dans la reprise du motif viril, mais déplacé dans un autre domaine de combat : la littérature. D'un côté, il s'agit de détruire par la subversion et, dans la prose des manifestes et des déclarations collectives, de faire corps et de porter une attaque collective, comme au front. D'un autre côté, on cherche à libérer des désirs inédits, à éclairer par le lyrisme les chemins de l'avenir, à établir les nouvelles modalités du corps à corps amoureux. Le surréalisme : difficile exercice de sublimation de la violence. Surréalisme de l'entre-deux : pris entre le marteau de la poésie action et la molle enclume d'une ouverture lyrique au miracle de ce qui vient, entre le texte d'intervention polémique et l'émerveillement intime du chant poétique, entre la virilisation qu'exigent les circonstances politiques et sociales et que commande l'état de la civilisation et une attention à la féminité qui ouvre d'autres espaces imaginaires et amorce d'autres pratiques. Surréalisme du trouble dans le genre, qui s'ouvre à la nouveauté et se crispe sur le passé et définit ainsi une posture inédite. Cette aventure porte bientôt ses fruits, lorsque, après 1945, l'activité surréaliste apparaît pour ce qu'elle est : une extension du domaine de l'intime en littérature (comme en politique d'ailleurs), avec la multiplication des femmes qui se réclament de lui et créent dans son orbite.

Guillaume Bridet Université Paris 13, Sorbonne Paris Cité, CENEL/PLÉIADE 